\title{
The Plasmodium falciparum var gene switching rate, switching mechanism and patterns of parasite recrudescence described by mathematical modelling
}

\author{
S. PAGET-MCNICOL ${ }^{1 *}$, M. GATTON ${ }^{1}$, I. HASTINGS ${ }^{2}$ and A. SAUL S $^{1} \uparrow$ \\ ${ }^{1}$ Malaria Biology Lab, Division of Infectious Diseases and Immunology, The Queensland Institute of Medical Research, \\ P.O. Royal Brisbane Hospital, Qld 4029, Australia \\ ${ }^{2}$ Liverpool School of Tropical Medicine, Pembroke Place, Liverpool L3 5QA, UK
}

(Received 27 May 2001 ; revised 19 September 2001 ; accepted 26 September 2001)

\section{SUMMARY}

Recrudescing Plasmodium falciparum parasitaemia is attributed to the switching of PfEMP1, a variant antigen family encoded by the var gene repertoire, and the host's immune response. We have developed a mathematical model which incorporates var gene switching, and variant specific, non-variant specific and non-specific immunity. By conducting a sensitivity analysis of the model we have defined the parameter limits which produce chronic and recrudescing infections. We explore 3 switching mechanisms : ordered, random and uncoupled switching. We show that if var genes switch on and off independently at variable rates through the repertoire a chronic clinical infection is predicted. The fastest switchingon rate that produces a chronic infection is $0.03 \%$ per generation. The model predicts that non-variant specific immunity plays an important role in reducing disease severity. This work illustrates the complex relationship between the malaria parasite and its host and shows that var gene switching at rates substantially slower than $2 \%$ are essential for parasite survival.

Key words: Plasmodium falciparum, var gene, switching rates, switching mechanism, disease severity, mathematical modelling.

\section{INTRODUCTION}

Parasite recrudescence and fever characterize Plasmodium falciparum malaria in non-immune humans (Collins \& Jeffery, 1999). The peaks of recrudescence, often separated by 3 -week intervals (Collins \& Jeffery, 1999), are attributed to the switching of $P$. falciparum encoded variant antigens exposed on the surface of infected erythrocytes (Biggs et al. 1991). Variant surface antigens have also been described in other human ( $P$. vivax and $P$. ovale), rodent $(P$. berghei and $P$. chabaudi) and simian $(P$. knowlesi, $P$. fragile and $P$. coatneyi) malaria species (Saul, 1999).

The $P$. falciparum erythrocyte membrane protein (PfEMP1) is a diverse set of variant proteins encoded by var genes (Baruch et al. 1995; Su et al. 1995). Each haploid malaria genome is thought to contain approximately 50 var genes (Su et al. 1995), although there may be fewer. Only 1 full-length var transcript is shown to be exposed as a PfEMP1 variant on each

* Corresponding author: Malaria Biology Lab, Division of Infectious Diseases and Immunology, The Queensland Institute of Medical Research, P.O. Royal Brisbane Hospital, Qld 4029, Australia. Tel: + 617 33620420. Fax: +61 7 33620104. E-mail:sarahM@qimr.edu.au $\uparrow$ Present address: Malaria Vaccine Development Unit, NIAID/LPD, National Institutes of Health, Rockville MD 20852, USA. erythrocyte surface (Chen et al. 1998; Taylor et al. 2000). The actual copy expressed by a cloned line can switch and in vivo, successive waves of parasites in a single host exhibit different surface antigen serotypes (Saul, 1999). In P. falciparum, expression of PfEMP1 is associated with the adherence of infected erythrocytes to endothelial cells and uninfected erythrocytes. Not only do different PfEMP1 variants have different serotypes, but the binding specificity of the infected erythrocytes also depends on the PfEMP1 type expressed (Biggs et al. 1992; Gardner et al. 1996). Thus, the expression of different PfEMP1 types may be associated with different aspects of severe malarial disease such as cerebral malaria (Carlson et al. 1990; Rowe et al. 1995), severe non-cerebral malaria (Raventos-Suarez et al. 1985) and infection of the placenta (Fried \& Duffy, 1996; Beeson et al. 2000).

The mechanism and regulation of PfEMP1 variant switching is not understood. It has been proposed that the cyclical expression of a particular surface antigen, the host's corresponding immune response to that antigen and the concurrent switching of the surface antigen serotype of a minor proportion of the parasite population is crucial to the establishment of a stable and chronic malaria infection (Saul, 1999).

The purpose of this paper is to present the results of a stochastic simulation model which investigates different mechanisms of switching, and the inter- 


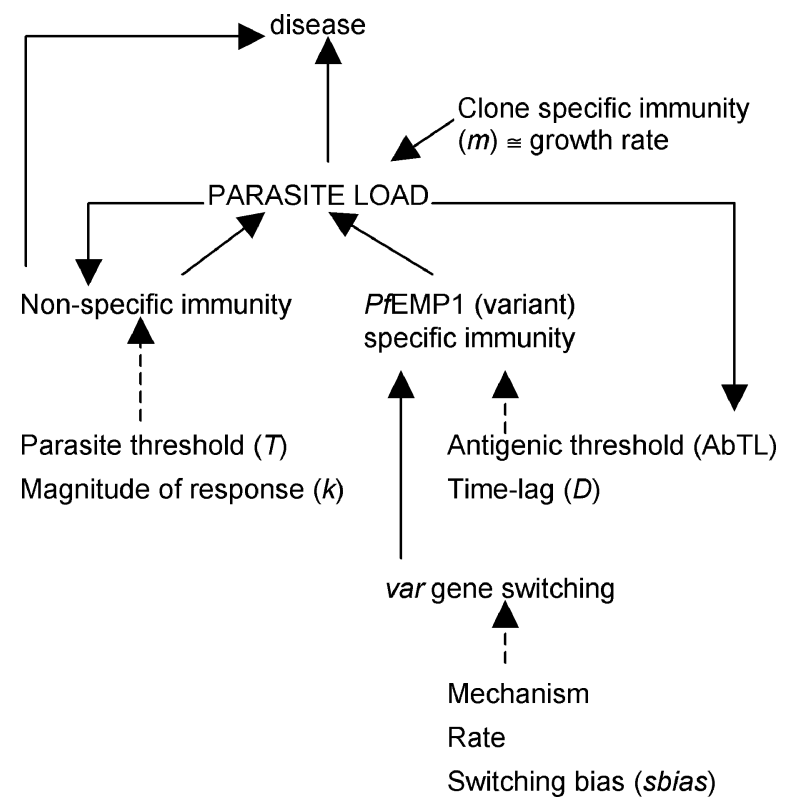

Fig. 1. The interaction of parameters which affect parasite load and disease. The parasite load (i.e. total number of parasites in the host) stimulates variant specific (VSI) and non-specific immunity (NSI). VSI is triggered once the antigenic threshold is exceeded and a time-lag occurs before anti-PfEMP1 antibodies become effective. The switching of var genes, which encode PfEMP1 variants, is determined by the mechanism and rate and influences the antibody response and thus the parasite load. NSI takes effect in the cycle that the threshold is exceeded. The magnitude of NSI is an indicator of the fever response and determines the degree to which the parasite load decreases. It is also associated with the clinical presentation of disease. Clone-specific immunity, against conserved determinants of a clonal population, is modelled by a decrease in the parasite growth rate, $m$, and is not influenced by the parasite load.

action between var gene switching and human immune responses. Intra-host models of malaria infection are not new (reviewed by Molineaux \& Dietz, 1999), but many are deterministic even though malaria infection is a stochastic process dominated by probabilistic events. Recently, Molineaux et al. (2001) reported a discrete-time simulation model incorporating a constant switching rate of $2 \%$ per generation, a switching probability that is modulated by the presence of variant specific antibodies and highly variable base line growth rates for each variant. The aim of their work was to accurately fit the model to a limited set of malariatherapy cases. In contrast, the relatively simple stochastic simulation mathematical model reported here is a generalized model which is not patient specific. We used our model in a sensitivity analysis to investigate the relationship between the PfEMP1 switching rate and parameters involved with the human immune response for several possible switching mechanisms. The model incorporates a rapidly acting, density-dependent, non-specific immunity, a specific immunity to individual $P f$ EMP1 variants, and a non-variant, specific immunity. The role of each of these types of immunity in the development of a chronic infection is also explored.

\section{METHOD}

The mathematical model is a stochastic model which describes var gene switching and the host's immune response to specific malaria antigens. Fig. 1 illustrates the interaction between the parameters of the model. The basic model incorporates non-specific immunity (NSI), PfEMP1 variant specific immunity (VSI) and non-variant specific immunity, referred to as 'clone specific immunity' (CSI). CSI may be against polymorphic antigens (such as the vaccine candidates merozoite surface protein-1 and apical membrane antigen-1) resulting in a different impact on different parasite clones, or CSI may be against common antigens resulting in pan-specific immunity. This distinction will be important when the model is extended to multiple infections. However, only clonal infections are considered here.

For simplicity we assume that each sporozoite which infects a naïve human host produces 40000 genetically identical merozoites, all expressing the same var gene. These merozoites are released into the blood-stream 6 days after infection (Coatney et al. 1971). During the erythrocytic cycle, on average 16 merozoites are released per schizont every $48 \mathrm{~h}$ (Coatney et al. 1971). Only a proportion of these merozoites invade new RBCs and survive to maturity; the other proportion are killed by clonespecific immunity (CSI). CSI is implemented as a reduction in the parasite growth rate from 12 -fold per cycle, the growth rate measured in P. falciparuminfected malaria naïve people (Cheng et al. 1997). In the model the effect of CSI remains constant throughout the infection. However, in vivo, the level of CSI may increase as the infection progresses.

Once the erythrocytic cycle begins, the parasites may switch variant antigens which are encoded by the var genes (Baruch et al. 1995). We assume each parasite has a repertoire of 50 var genes ( $\mathrm{Su}$ et al. 1995). Three different var gene switching mechanisms are investigated and are based on experimental observations of clonal antigenic variation in malaria parasites. The first switching mechanism modelled is sequential and regulated: a single var gene can only switch to the next var gene in the series. The probability of switching from var $i$ to var $j$ is zero, except when $j=i+1$ in which case the probability is 1 (Table 1 ). The switching rates for each var gene in the repertoire can be either equal, randomly selected within a specified range or linearly decreasing. This ordered mechanism mimics the sequential appearance of $P$. fragile variant antigens, where each variant corresponds to a new wave of parasitaemia (Handunnetti, Mendis \& David, 1987). 
Table 1. The probability of switching from one var gene to the next var gene according to the switching mechanisms

(The switching mechanisms modelled were ordered, random and uncoupled. Variations of the mechanisms incorporated a switching bias (sbias) which created a series of non-uniform probabilities and resulted in the expression of fast (major) and slow (minor) switching var genes.)

\begin{tabular}{ll}
\hline \hline Switching mechanism & Probability, $p$, of switching from var $i$ to $\operatorname{var} j(p[i, j])$ \\
\hline 1. Ordered & $p[i, j]=1$, if $j=i+1$ \\
& $p[i, j]=0$, if $j \neq i+1$ \\
2. (a) Random-uniform & $p[i, j]=1 / 49$ \\
(b) Randon-non-uniform & $p[1,1]=1$ \\
& $p[1, j]=p[1, j-1] /$ sbias for $j=2 \ldots 50$, \\
& $z=\sum_{j=1} p[1, j]$, \\
(i) next type & $p[i, j]=p[1, j] / z$ \\
(ii) next and last & $p[i, j]=p[1,51-j] / z$ for $j=1 \ldots i-1$ and, \\
& $p[i, j]=p[1, j-i+1] / z$ for $j=i \ldots 50$ \\
3. Uncoupled & $p[1]=1$ \\
& $p[i]=p[i-1] /$ sbias \\
& $z=\sum_{i=1}^{50} p[i]$ \\
(i) switching on & $p_{\text {on }}[i]=p[i] / z$ \\
(ii) switching off & $p_{\text {off }}[i]=p[i] / z$ \\
\hline
\end{tabular}

Table 2. The range of parameter values simulated which control the parasite population growth

(The upper and lower limits for each variable were determined by conducting 2000 simulations through the range of values presented, while the values of the other variables were kept constant.)

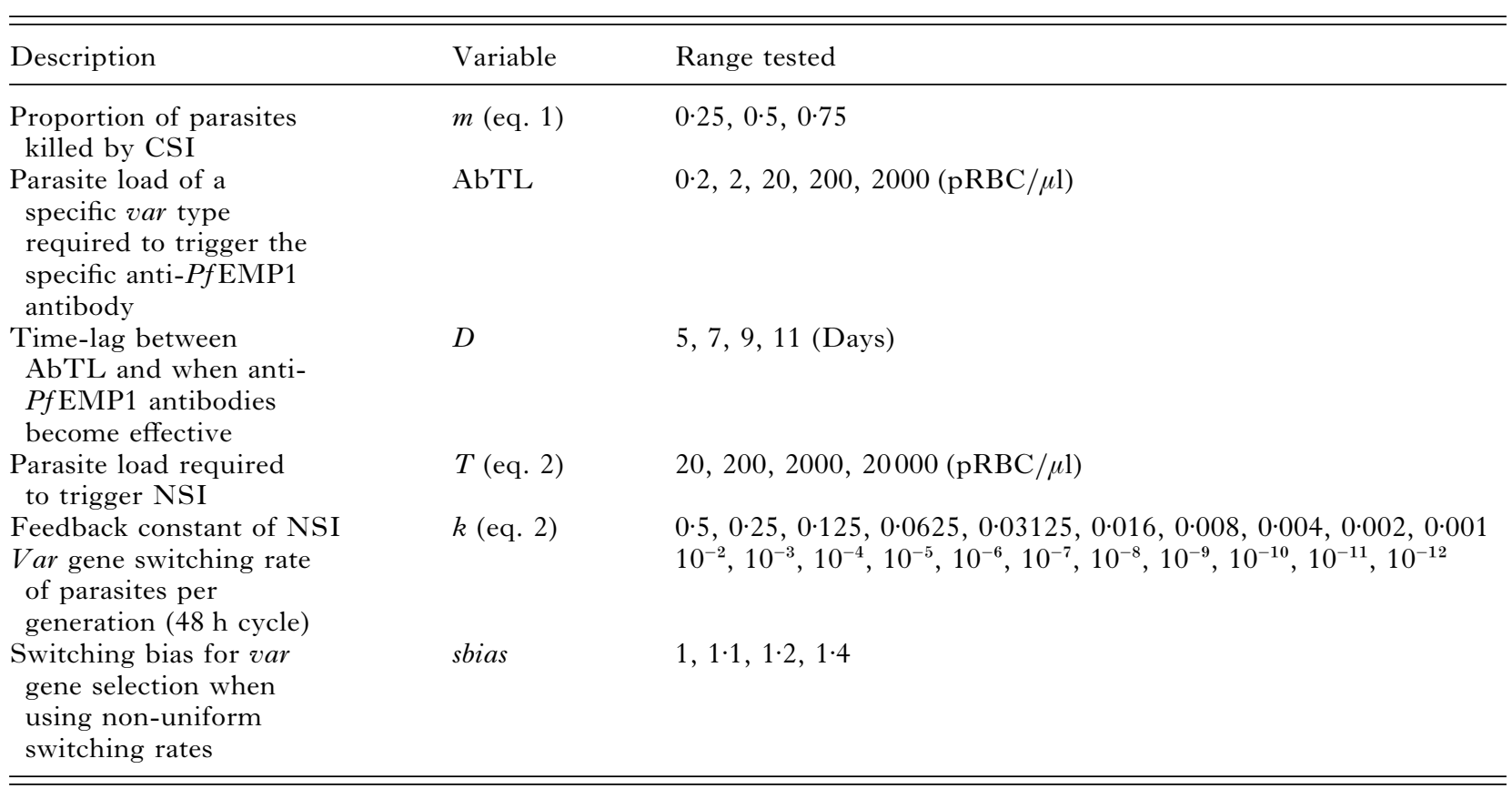

The second mechanism modelled allows a particular var gene to switch to any other var gene in a random manner. The basic mechanism ensures that each var gene has an equal chance of switching on so that the probability of switching from var $i$ to var $j$ is $1 / 49$. Two variations of this mechanism incorporate a switching bias (sbias) (Table 2) which creates a repertoire of non-uniform switching probabilities. The first variation ensures that the probability of switching is dependent on the next var gene to be expressed (Table $1-$ eq. 2b(i)). The second variation ensures that the probability of switching is dependent on the current var gene expressed and the next to one to be switched on (Table $1-$ eq. $2 \mathrm{~b}(\mathrm{ii})$ ). This variation also allows backward switching to a previously expressed var gene. These variations of the random mechanism model the expression of major and minor variant serotypes in $P$. chabaudi infections, where several unique variants were shown to be present sim- 
ultaneously during a wave of parasitaemia (Brannan, Turner \& Phillips, 1994).

The third mechanism, referred to as uncoupled switching, allows an expressed var gene to switch off independently of another var gene switching on, such that var $i$ is switched off independently of var $j$ switching on (Table 1 - eq. 3). Thus a parasite may have 0,1 or 2 var genes expressed at any one time. The on and off switching rates can be equal or unequal, and either uniform (sbias $=1)$ or nonuniform (sbias $>1$ ) through the var gene repertoire. This mechanism models the loss and then reexpression of variant antigens on infected erythrocytes (reviewed by Newbold et al. 1999). In the model, parasites which do not express any var genes are killed (this simulates the action of the spleen).

Variant specific antibodies develop once the number of parasites expressing a particular var antigen reaches a defined antigenic threshold (AbTL). To incorporate the time required for antibody development in the host, a time-lag must be exceeded before anti-PfEMP1 antibodies take effect. The number of parasites surviving CSI and variant specific immunity (VSI) follows a binomial distribution (eq. 1).

$X_{i} \approx B i\left\{X_{i},(1-m) K\left(X_{i}\right)\right\}$,

where $X_{i}$ is the number of parasites expressing var $i$, $m$ is the probability of a parasite being killed by clone-specific immunity and $K\left(X_{i}\right)$ is the probability of a parasite expressing var $i$ surviving the specific immunity against var $i$.

The model assumes that non-specific immunity (NSI) becomes functional when the total number of parasites exceeds a defined parasite threshold level (Collins \& Jeffery, 1999; M. Gatton, personal communication). The degree of parasite killing invoked by NSI is a function of the difference between the parasite load and the parasite threshold. In preliminary modelling a number of functions were considered with an exponential function consistently giving the most realistic patterns of parasitaemia. For simplicity, it is assumed that the parasite threshold and feedback constant, $k$, are constant throughout an infection, although in vivo, one or both may change during the course of an infection. Unlike VSI, the effect of NSI is immediate. The number of parasites surviving NSI is described using a binomial distribution (eq. 2).

$X_{i} \approx B i\left(X, e^{-k(X-T) / T}\right)$,

where $X$ is the total parasite load, $T$ is the parasite threshold that stimulates NSI and $k$ is the feedback constant $(0<k \leqslant 1)$. The probability of parasites surviving NSI is inversely proportional to $k$. It follows that an increase in the value of $k$, decreases the parasite load through non-specific immune killing.

The total number of parasites, the number of parasites expressing each var gene, the day the variant expressing a particular var gene reaches its maximal parasitaemia and the maxima and minima of each parasitaemia peak are tracked and recorded throughout the simulation period of 200 days. Each simulation of the model produces a data output and a graph of parasite recrudescence over time with a fever indicator proportional to the NSI response. A sensitivity analysis of 2000 simulations on each parameter value (Table 2) was conducted to determine parasitaemia patterns comparable with at least part of the parasite profiles recorded from 148 neurosyphilitic patients treated with $P$. falciparum who were not given anti-malarial treatment to modify or eliminate the primary episode of parasitaemia (Collins \& Jeffery, unpublished data). Parameter sets which were able to produce peak parasite loads between $10^{3}$ and $10^{5}$ parasitized $\mathrm{RBC} / \mu 1$ blood (pRBC/ $\mu \mathrm{l}$ ), an infection exceeding 50 days, a fever response and recrudescing parasitaemia with an approximate 3 -week interval between peaks were considered as realistic patterns of malaria infections. The switching rate reported from the sensitivity analysis is the rate multiplied by the highest probability of switching within the repertoire.

\section{RESULTS}

The stochastic nature of the model produces variation in graphical outputs when the same set of parameter values are used thus reproducing the individuality of malaria infections. Fig. 2 compares a sample of the clinical data (Collins \& Jeffery, unpublished data) with a model output.

\section{Clone-specific immunity}

For all mechanisms of switching, chronic patterns of parasite recrudescence reaching levels between $10^{3}$ and $10^{5} \mathrm{pRBC} / \mu 1$ were achieved with CSI resulting in growth rates of 8 -fold per cycle or above (Fig. 3). When CSI caused the growth rate to fall to 4 -fold per cycle, patent chronic periodic infections could only be achieved when the threshold required to trigger VSI was greater than $2 \mathrm{pRBC} / \mu 1$. However, the patterns of parasitaemia generated did not represent the other features of a chronic clinical infection.

\section{Non-specific immunity}

In the model, 2 factors determine the effects of NSI : the parasitaemia which triggers NSI and the size of the feedback constant $(k)$. Realistic infection patterns were obtained when the parasite threshold was between 200 and $2000 \mathrm{pRBC} / \mu$ l. Deterministically, there is a simple relationship between the parasite growth rate, the parasite threshold $(T)$, the feed back 

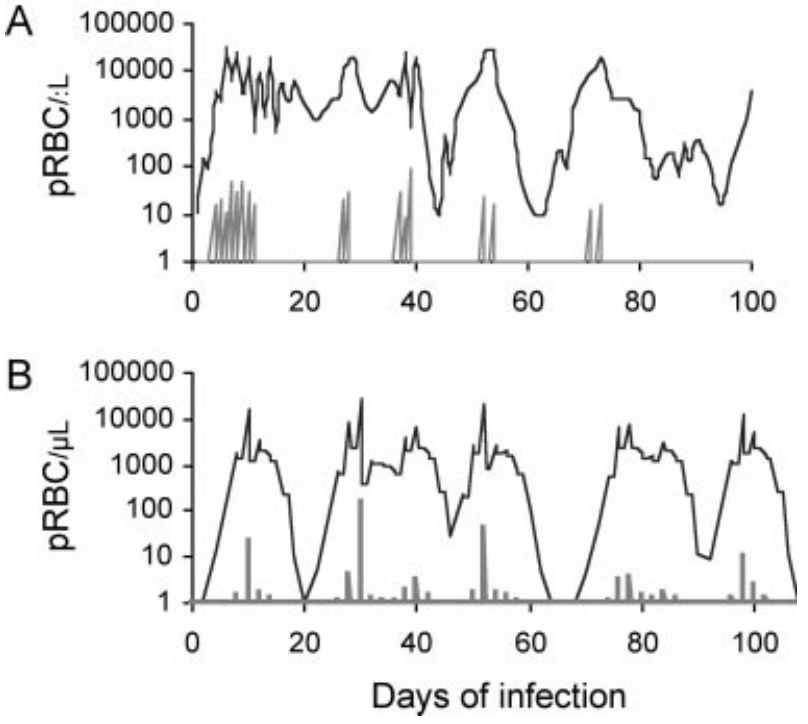

Fig. 2. The patterns of parasite recrudescence (top curve) and fever indicator (spikes) in a Plasmodium falciparum infection. (A) Clinical case of patient infected with the El Limon strain of $P$. falciparum (Collins \& Jeffery, unpublished data). (B) Model output of uncoupled and non-uniform switching between var genes: fastest on rate $=10^{-8}$ and fastest off rate $=10^{-2}$ switching parasites per generation. The thresholds for VSI and NSI are $2 \mathrm{pRBC} / \mu 1$ and $200 \mathrm{pRBC} / \mu 1$, respectively, with a low NSI feedback constant $(k=$ $0 \cdot 03125)$. NSI, in terms of the fever response, causes sharp oscillating peaks within each recrudescence. Parasite recrudescences begin on Days 20 and 68 and occur after a genetic bottleneck which results from the effect of VSI.

constant $(0<k<1)$ and the level at which the parasitaemia stabilizes (ignoring the effect of VSI). This relationship is described by:

parasitaemia $=(T / k) \ln ($ growth rate $)+T$.

For a large feedback (as $k \rightarrow 1$ ) the average parasitaemia stabilizes close to, but above, the parasite threshold, $T$, but the instantaneous values show considerable variation. Since NSI is only applied once per cycle as the schizonts rupture, the feedback loop is destabilized, and a large feedback constant causes a big spike of NSI. This is depicted in the fever response and results in a high proportion of parasites being killed. The parasite population requires several cycles to again reach the parasite threshold, at which time the NSI is triggered and the process repeats itself. This behaviour resembles the early stages of a malaria infection with high fever spikes and a degree of chaotic behaviour in both the parasite levels and fever response. When the feedback is small $(k \rightarrow 0, k \neq 0)$, the parasitaemia stabilizes at a level much greater than the parasite threshold, resulting in small and consistent NSI responses per cycle. This resembles the later stages of a malaria infection. Irrespective of the amount of feedback, the deterministic analysis indicates that
NSI alone is not enough to control parasitaemia since the equilibrium is always above the fever threshold. For any feedback constant value, the size of the fever response (and presumably the fever experience in vivo) is closely related to the parasite growth rate and thus the level of CSI. By increasing CSI, the growth rate decreases causing a substantial decrease in the size of the parasite spikes due to NSI and the size of the fever response (Fig. 3).

\section{Var-specific immunity}

Realistic patterns of infection occur when the antigenic threshold is between 2 and $200 \mathrm{pRBC} / \mu 1$. When the threshold is lower $(0 \cdot 2 \mathrm{pRBC} / \mu \mathrm{l})$ the infection does not exceed 20 days. There is also no fever response, since the parasite population is killed by variant specific antibodies before the fever threshold is triggered and before any parasites switch to a new unexpressed var gene. A higher threshold of $2000 \mathrm{pRBC} / \mu \mathrm{l}$ results in the parasitaemia reaching $10^{6} \mathrm{pRBC} / \mu \mathrm{l}$. This is equivalent to a $20 \%$ parasitaemia which would kill the patient since, unlike the NSI response against the parasite and its toxins, there is a delay between antibody production and release in response to variant antigens.

We investigated 5, 7, 9 and 11 day delays of variant specific antibody killing. Irrespective of the switching rate and the level of NSI, low parasitaemia levels and regular recrudescence patterns were predicted with the 5 day time-lag. This reflected dominant antibody control. A 9 or 11 day time-lag resulted in a pattern of parasitaemia consistently above the detection threshold of $10 \mathrm{pRBC} / \mu 1$, reflecting weak antibody control. A 7 day time-lag produced the most realistic recrudescing patterns of parasitaemia with peaks and troughs above and below the detection threshold.

\section{Interactions of the immune response}

The parasite load is dependent upon the ratio of the VSI and NSI thresholds. For a chronic and periodic infection to occur, the VSI antigenic threshold must be at least 10-fold lower than the NSI parasite threshold. A ratio less than 10 results in the parasitaemia being controlled by NSI alone which is depicted by sharp oscillating peaks. Thus the parasitaemia never falls below the detection limit and the classical patterns of recrudescence do not occur. A maximum difference of 1000-fold is possible (e.g. VSI threshold is $2 \mathrm{pRBC} / \mu \mathrm{l}$ and NSI threshold is $2000 \mathrm{pRBC} / \mu \mathrm{l})$. A ratio greater than this results in no fever response with the parasitaemia controlled by VSI before the NSI threshold is reached. Therefore NSI plays a significant role in controlling parasite levels during the antibody timelag period but alone cannot control the parasitaemia. 
Parasite population growth rate

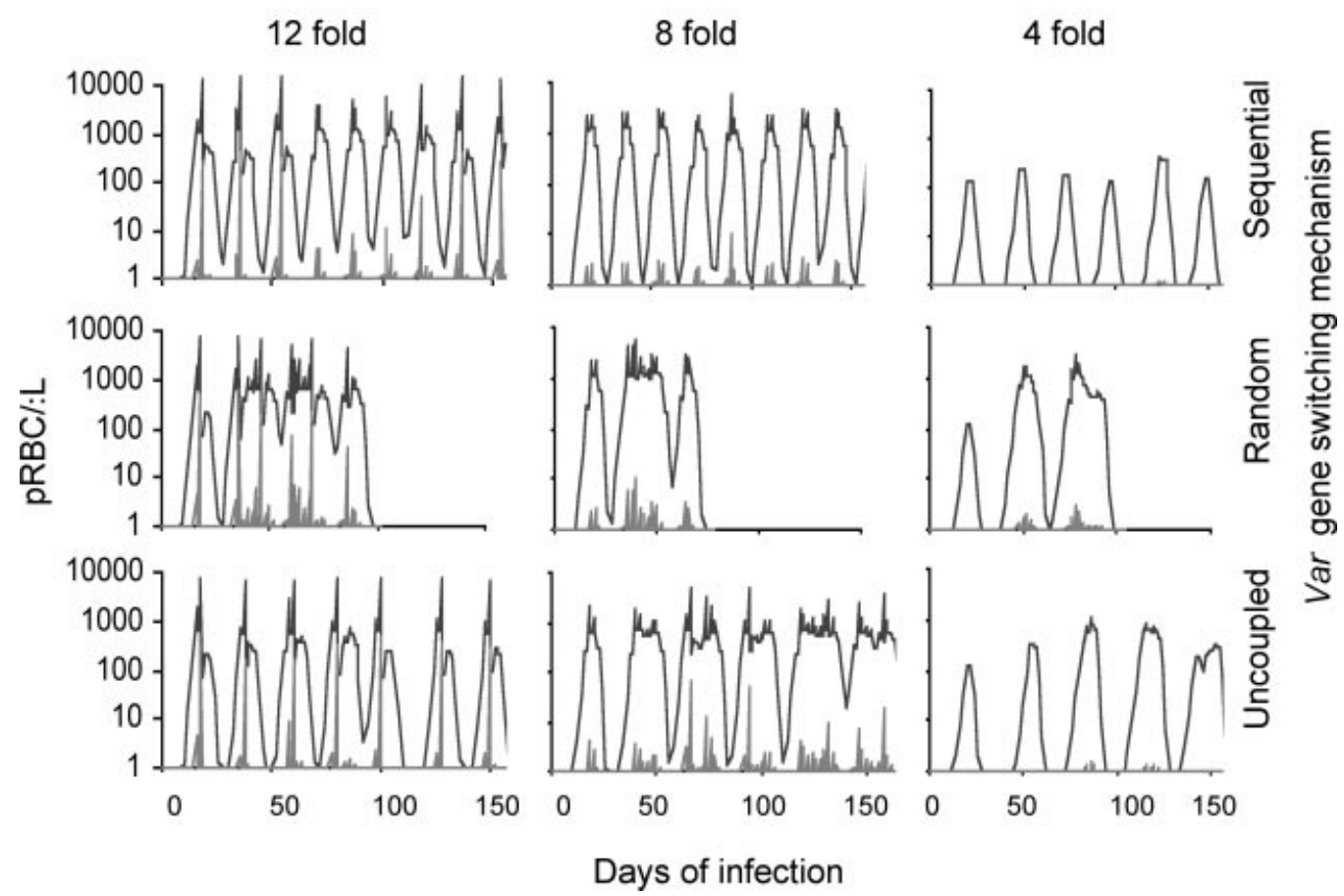

Fig. 3. The decrease in fever response (disease severity) associated with a decrease in the parasite growth rate (modelled by CSI). Top curve represents the parasitaemia and the spikes represent the fever indicator. For all graphs the thresholds for VSI and NSI are $2 \mathrm{pRBC} / \mu 1$ and $200 \mathrm{pRBC} / \mu 1$, respectively, with a medium NSI feedback constant $(k=0.0625)$ and a slow switching on rate of $10^{-8}$ for the sequential and random models and an on rate of $10^{-6}$ for the uncoupled model.

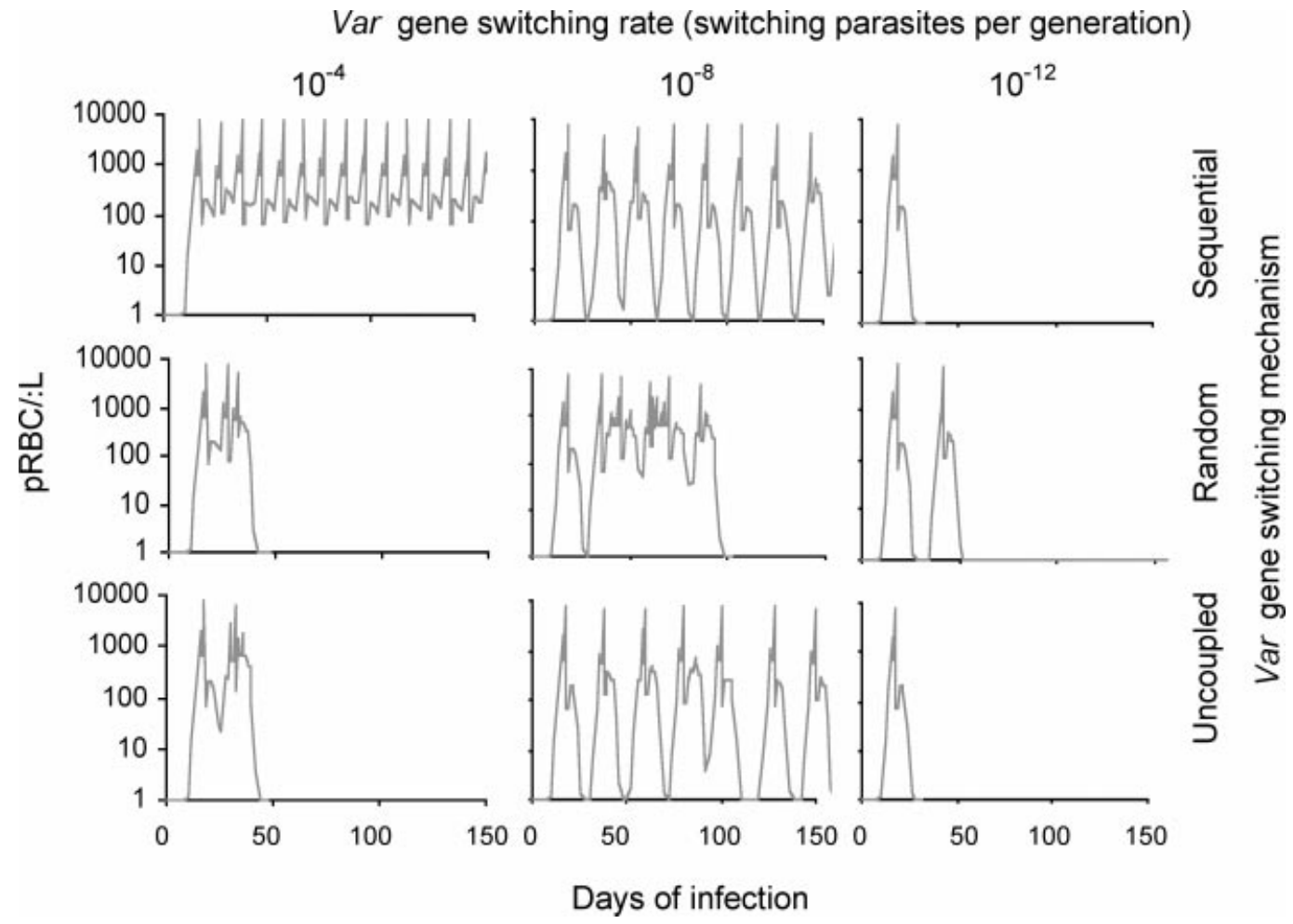

Fig. 4. The patterns of parasite recrudescence resulting from fast, medium and slow var gene switching rates. For all graphs the thresholds for VSI and NSI are $2 \mathrm{pRBC} / \mu 1$ and $200 \mathrm{pRBC} / \mu 1$, respectively, with a medium NSI feedback constant $(k=0.0625)$ and a level of CSI which allows a 12 -fold parasite population growth rate. 


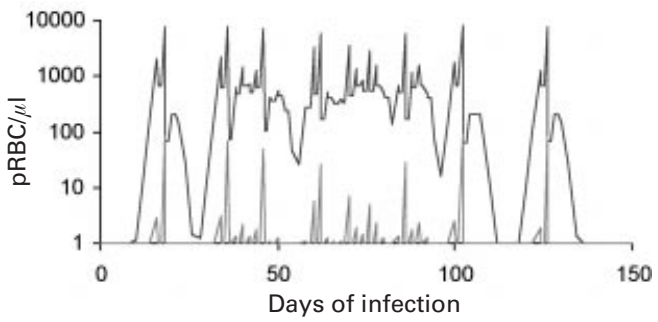

Fig. 5. Patterns of parasite recrudescence (top curve) produced by non-uniform random switching model. The spikes represent the fever indicator. Var gene switching is $10^{-8}$ switching parasites per generation and is dependent upon the next var gene to be expressed. The thresholds for VSI and NSI are $2 \mathrm{pRBC} / \mu 1$ and $200 \mathrm{pRBC} / \mu 1$ respectively, with a medium NSI feedback constant $(k=0.0625)$ and a level of CSI resulting in a 12 -fold growth rate.

\section{The var gene switching mechanism}

Using the parameter limits determined by the sensitivity analysis, the sequential, random and uncoupled switching mechanisms were evaluated to determine which produced the most realistic patterns of parasitaemia and fever.

The sequential switching of var genes using any of the rate variations, results in regular patterns of recrudescence with little chaotic behaviour. When switching rates decrease through the var gene repertoire, periodic recrudescences with approximate 21-day periodicity between peaks occur. These regular patterns resemble the middle phase of an infection (Fig. 4, top middle panel).

Random switching of var genes at a uniform rate can not predict chronic infections (Figs 3 and 4, middle panels). However, when switching is random and non-uniform, with some var genes in the repertoire having a higher probability of switching relative to the other var genes, the characteristic patterns of recrudescence can be predicted at slow switching rates (Fig. 5).

Realistic patterns of recrudescence are predicted by the uncoupled on and off switching of var genes at equal (Fig. 3, bottom middle and left panels) and unequal switching rates (Fig. 2B). Fig. 6 illustrates the expression of 11 different var genes during the first 150 days of an infection, showing that with the uncoupled mechanism only a small subpopulation of parasites, usually at an undetectable level, will express 2 var genes simultaneously. The switching off of one of these genes allows these variants to recrudesce and maintain the infection.

\section{The var gene switching rate}

The switching rate is modelled as the rate multiplied by the probability of switching and this switching rate is reported as the fastest switching var gene in the repertoire. For all switching mechanisms fast switching rates of $10^{-2}$ to $10^{-3}$ switching parasites per generation (equivalent to $1 \%$ and $0 \cdot 1 \%$ ) result in a non-recrudescing acute parasitaemia of less than 50 days. This fast switching exhausts the var gene repertoire resulting in short infection periods.

For sequential switching of var genes with decreasing rates through the repertoire, the upper and lower limits on the fastest rate are $10^{-7}$ to $10^{-9}$ switching parasites per generation respectively. The upper and lower limits for purely random var gene switching are lower at $10^{-9}$ and $10^{-12}$ switching parasites per generation respectively. When a non-uniform random switching mechanism is used, the respective upper and lower limits of the fastest rate increase to $10^{-6}$ and $10^{-11}$ switching parasites per generation.

The upper and lower parameter limits on the switching-on rate for the basic uncoupled mechanism are $3 \times 10^{-5}$ and $3 \times 10^{-7}$ switching parasites per generation. Using the switching bias to create non-uniformity of the total switching rate through the repertoire predicts higher upper and lower parameter limits for the fastest switching-on rates of var genes of $3 \times 10^{-4}$ and $3 \times 10^{-6}$ switching parasites per generation respectively. For both cases, the upper and lower parameter limits on the switchingoff rate equals those on the switching-on rate.

When the on and off switching rates are unequal an interesting balance occurs: a fast on rate predicts chronic patterns of parasitaemia only when the off rate is slow. Likewise, a slow on rate couples with a fast off rate. When the switching through the repertoire is uniform but the switching-on and -off rates are unequal, the upper and lower limits on the switching-on rate are $10^{-4}$ and $10^{-9}$ switching parasites per generation respectively. The upper and lower limits on the switching-off rate are $10^{-2}$ and $10^{-8}$ switching parasites per generation respectively. A fast on rate of $10^{-4}$ couples with slow off rates of $10^{-7}$ and $10^{-8}$ and a slow on rate of $10^{-9}$ couples with fast off rates of $10^{-2}$ and $10^{-3}$ switching parasites per generation. When the switching through the repertoire is non-uniform, the upper and lower limits on the switching-on and-off rates are the same as those when switching through the repertoire is uniform, indicating that the fastest possible respective switching-on and -off rates are $10^{-4}$ and $10^{-2}$ switching parasites per generation.

\section{DISCUSSION}

Malaria is a complex disease which requires a delicate balance between parasite growth and host immunity to enable survival of both the parasite and its host. The variability and switching of PfEMP1 antigens is a major factor in the survival of the parasite population until transmission. The host produces a strong immune response against PfEMP1 and other polymorphic antigens, as well as a non-specific response in an attempt to destroy the parasite population. Such complexity and interactions are 

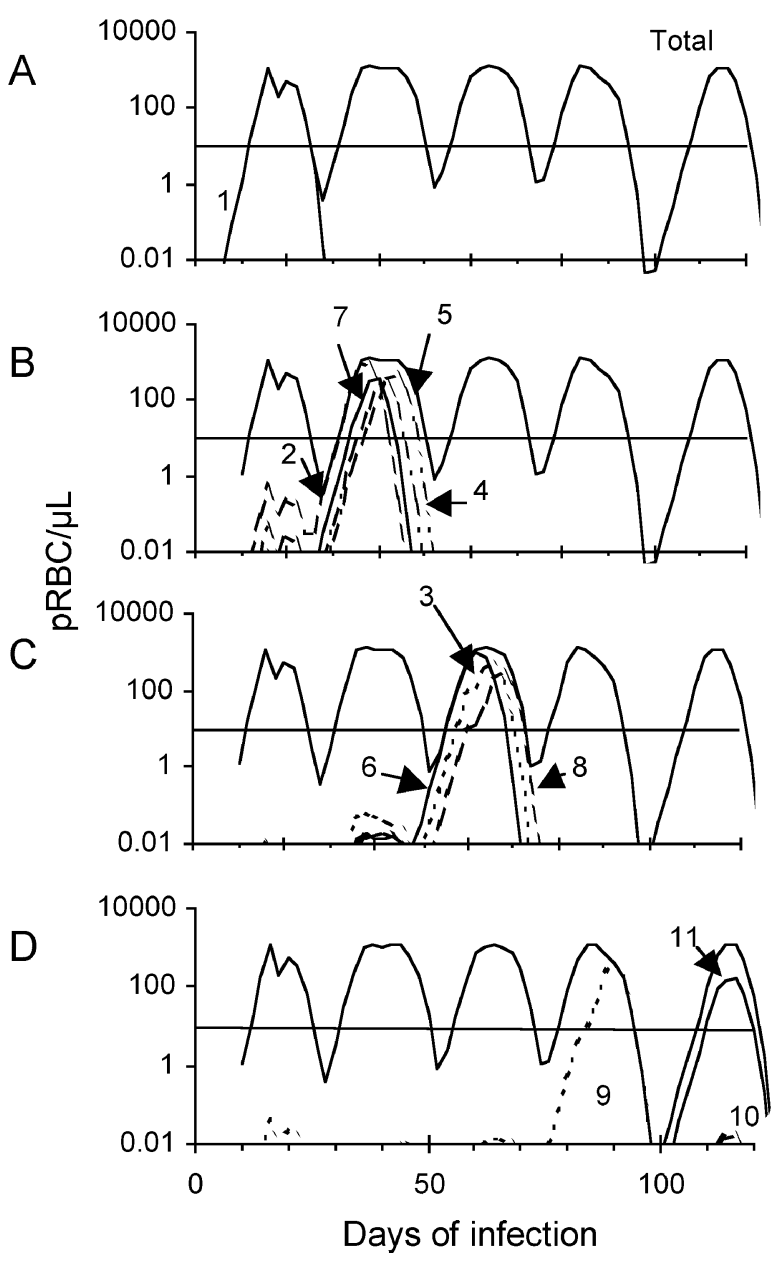

Fig. 6. The 4 panels represent a single Plasmodium falciparum malaria infection modelled by the uncoupled switching of var genes. The line represents the detection limit of $10 \mathrm{pRBC} / \mu \mathrm{l}$. Each panel shows a wave or waves of parasitaemia consisting of different types of expressed var genes at different parasite levels during the course of infection. A wave of parasitaemia may consist of predominantly 1 expressed var gene product (e.g. the first parasite peak) or many expressed var gene products (e.g. the second parasite peak $=$ first parasite recrudescence). The uncoupled on and off switching of var genes allows a small subpopulation of parasites to express 2 var gene products. The size of this subpopulation depends upon the switching-on and -off rates. These doubly expressed variant parasites are susceptible to the antibodies against either of the expressed var genes. For example, in the first peak the dominant variant expresses var 1 (A) and the minor variants of interest express vars 1 and 2, 1 and 4, 1 and 5 , and 1 and 7 (B) and 1 and 9 (D), where vars 5 and 7 are below $0.01 \mathrm{pRBC} / \mu 1$ in the first peak. Antibodies against the expressed var 1 gene product cause the minor populations to follow the same pattern of parasitaemia as that of the major population. Some parasites in the minor populations are able to survive the VSI against the var 1 gene product by switching var 1 off. These parasites are then able to recrudesce whereas the major population expressing only var 1 falls below 0.001 pRBC/ $\mu$ l before Day 30 (A). Many minor populations will remain undetectable until later in the infection (e.g. var genes 3, 6 and 8 which surpass the difficult to investigate experimentally but can be explored with mathematical models. Our model of a malaria infection investigates the complex patterns of parasite recrudescence in an initially non-immune patient and provides limits on the thresholds governing host immunity and on the range of var gene switching rates according to specific switching mechanisms.

The model presented here incorporates the successive switching of individual parasite var genes, the production of variant specific antibodies and a negative feedback loop of the NSI response which kill a proportion of all parasites. The mathematical model described by Molineaux et al. (2001) also incorporates these features but many are based on assumptions which make it biologically unlikely. First, the switching from var $i$ to var $j$ requires immune modulation, such that the actual probability of switching to var $j$ is dependent upon the presence of variant specific antibodies against other expressed var gene products. Once the new var gene is switched on, the switching probability between other var genes increases. Our model incorporates a far more simplistic and biologically plausible switching methodology where the switching probabilities are inherent and are therefore not modulated by the immune response. P. falciparum variants switch in vitro where there is no immune pressure, thus supporting an inherent switching mechanism (Biggs et al. 1992; Roberts et al. 1992). In vivo, the expressed var gene products are undoubtedly modulated by the host's immune response resulting in selection pressure against the PfEMP1 variant antigens and thus against the specific variant parasite subpopulations (Bull et al. 1998; Iqbal et al. 1997; Saul, 1999).

Second, Molineaux et al. (2001) fitted the model to allow each PfEMP1 variant to have different growth rates. However, their model described a monoclonal infection in which all parasites are genetically identical other than the expressed var gene. The authors justified the use of different variant growth rates from their estimation of a broad range of initial growth rates in the acute phase of parasitaemia. However, there are many reasons why these initial growth rates vary, without invoking different growth rates due to different var gene expression. For example, this range of growth rates comes from single observations in different people who were infected with 1 of 3 different parasite lines. Even when the same line was used, the inocula of these lines was associated with different passage histories. There is also a substantial error in measuring initial

detectable threshold in peak 3, var 9 in peak 4 and var 11 in peak 5), and others always remain at very low undetectable levels throughout the whole infection (e.g. var gene 10 shown in $\mathrm{D}$ ). 
growth rates when the parasitaemia is low, and, in the method used by Molineaux et al. (2001) at least part of that parasite growth occurred during a nonexponential growth period which was probably due to the effect of non-specific immunity. Although Molineaux et al. (2001) argued that the survival of the schizont may depend upon the var gene expressed and the subsequent adherence properties of the PfEMP1 antigen, we can find no direct evidence to show that at the very low initial parasitaemia cytoadherence is limiting. There is, however, direct evidence that the same parasite inoculum produces initial growth rates which vary from person to person (Lawrence et al. 2000), albeit at a considerably lower variation than that required by the Molineaux model. As we show in this paper, such assumptions are not required to generate an adequate model of malaria.

In contrast to the Molineaux model, the model presented here uses simple equations that require few input parameters to describe the various immune responses. Furthermore the model is generalized in that no individual specific data are required, making the model applicable to many situations. This relative simplicity does not appear to compromise the reality of the model output since it is able to explain the many features of a malaria infection. We have shown that var genes switching on independently of other var genes switching off produces realistic patterns of parasite recrudescence. Compared to the sequential and random mechanisms, uncoupled switching may be the most biologically plausible mechanism for the control of gene expression. Supporting evidence for the uncoupled mechanism comes from several experimental observations of new variants arising in animal models: the expression of 'variant-negative' Plasmodium infected RBCs from a cloned population, which later become 'variant-positive' (reviewed by Newbold et al. 1999); the ordered expression of variants in successive waves of parasitaemia (Handunnetti et al. 1987); and the expression of major and minor variants and the detection of several unique variants present simultaneously during a wave of parasitaemia (Brannan et al. 1994). The uncoupled mechanism allows either 0,1 or 2 var gene products to be expressed on the surface of an infected RBC, a unique concept for var gene expression. The majority of parasites will express a single var gene. However, a small proportion of the population may not express any var genes. In vivo, these parasites are more likely to be removed from the circulation by the spleen (Saul, 1999). Another small proportion may express 2 different var genes, eliciting a stronger anti-PfEMP1 response and thus helping to regulate parasite growth. At first it appears that the uncoupled mechanism contradicts the experimental evidence that only 1 var gene product is expressed (Chen et al. 1998; Taylor et al. 2000). However, only a very small proportion of the parasites express 2 var genes $(\ll 1 \%)$ and it would not be expected that this subpopulation would be detected in bulk cultures (Taylor et al. 2000), and highly unlikely that a doubly expressing parasite would be selected for single cell micro-manipulation (Chen et al. 1998). The experimental evidence does not discount the proposed uncoupled switching mechanism, but such experimental techniques are not yet sensitive enough to detect the subpopulation should it exist.

For each mechanism, when each var gene in the repertoire was allowed to switch on at rates greater than $0.1 \%$ per generation, chronic patterns of parasite recrudescence and fever could not be predicted. This result contradicts the fast antigenic switching rate of $2.4 \%$ per generation reported for $P$. falciparum variants in vitro (Roberts et al. 1992). However, this fast rate was based on 11 out of 21 clones switching after 30 generations (Roberts et al. 1992), and represents the sum of all variants that had switched agglutinating characteristics relative to the parent. It is not the rate at which a single variant appears nor the rate at which a var gene is expressed. Assuming that the antigenic variation was due solely to changes in the $P f$ EMP1 phenotype, then the rate that a single variant may have switched on could be as low as $0 \cdot 16 \%\left(1-\exp \{\ln (20 / 21) / 30\}=1 \cdot 6 \times 10^{-3}\right)$. Our modelling predictions do not discount the fast switching rates estimated both in vitro (Roberts et al. 1992) and in vivo (Brannan et al. 1994), but show that an entire var gene repertoire consisting of fast switching var genes would be exhausted within the first few weeks of an infection. Such a repertoire is therefore not compatible with chronic and stable recrudescing parasitaemias. Molineaux et al. (2001) produced similar results using a switch rate of $2 \%$ per generation with a median of 45 variants being expressed in the best runs of their model. However, the problem of exhausting all of the var genes early in the infection was resolved in their model by allowing each variant to have a different multiplication rate.

A var gene repertoire in which a minority of genes switch at a fast rate, creating the characteristic acute phase of parasitaemia, and the remainder of var genes switch at much slower rates, produces patterns of recrudescing and chronic parasitaemia. This rational is supported by experimental observations which show variable antigen types of $P$. chabaudi switching at differential rates (Brannan et al. 1994). Indeed Roberts' data (Roberts et al. 1992) also shows that 2 out of the 11 clones switched to the same variant type, implying that the switching probability to this variant may have been higher than to any other variant.

A repertoire of var genes, in which there is only a small probability that some var genes will switch on and be expressed due to slow switching rates, may also explain why severe malaria is a relatively rare 
event considering the millions of people infected with malaria annually. This supports the proposal that severe malaria may be caused by only a small proportion of the total var gene repertoire (Roberts et al. 1993).

A close interaction between the thresholds governing NSI and VSI and the var gene switching rate was revealed by the modelling. To sustain an infection, Saul (1999) proposed that the switching rate should be comparable with the rate at which immune responses are generated. We extend this proposal and suggest that the average switching rate of each var gene repertoire should be inversely related to the parasite load which induces a variant specific immune response. Modelling predictions also show that if the overall parasite growth rate is reduced to approximately 4-fold per cycle by CSI, then the severity of disease, illustrated as the fever response, is markedly reduced. This has practical implications for an anti-disease vaccine and shows that a vaccine which boosts CSI would only have to be $66 \%$ effective to have an impact on malaria morbidity and the associated economic losses due to malaria illness. Slow switching rates of PfEMP1 variants would enable such a vaccine to remain effective.

The results of the modelling indicate that to generate a chronic infection with parasite recrudescences, a complex and dynamic interaction between VSI, CSI, NSI and the switching rate of var genes exists. Var gene switching is a stochastic process giving rise to phenotypic heterogeneity and we propose a mechanism of uncoupled switching to explain this heterogeneity and the patterns of chronic parasitaemia. We have presented a robust and biologically plausible model of a malaria infection. This basic model can be extended to investigate many other areas of a clinical infection. These include the primary and secondary immune responses to conserved regions on multi-variant gene families in addition to the variant regions modelled, the change in the thresholds governing VSI, CSI and NSI during the course of infection which would likely influence disease severity, and the probability of drug-resistant mutants surviving to transmission with or without anti-malarial intervention.

The work was funded by the National Institutes of Health (grant no. \#AI47500-02) and the author SP was funded by an Australian Postgraduate Award through the University of Queensland. Thank you also to Dr Qin Cheng and Professor Bill Hogarth for their valuable comments.

\section{REFERENCES}

BARUCh, D. I., PASlOSKe, B. L., SINGH, H. B., BI, X., MA, X. C., FEldman, M., TARASChi, T. F. \& HOWARD, R. J. (1995). Cloning the Plasmodium falciparum gene encoding $P f$ EMP1, a malarial variant antigen and adherence receptor on the surface of parasitized

human erythrocytes. Cell 82, 77-87.

BEESON, J. G., ROGERSON, S. J., COOKE, B. M., REEDER, J. C., CHaI, W., LAWSON, A. M., MOLyneux, M. E. \& BROWN, G. v. (2000). Adhesion of Plasmodium falciparuminfected erythrocytes to hyaluronic acid in placental malaria. Nature Medicine 6, 86-90.

BIGGS, B. A., ANDERS, R. F., DILlON, H. E., DAVERN, K. M., MARTIN, M., PETERSEN, C. \& BROWN, G. V. (1992). Adherence of infected erythrocytes to venular endothelium selects for antigenic variants of Plasmodium falciparum. Fournal of Immunology 149, 2047-2054.

BIGGS, B. A., GOOZE, L., WYCHERLEY, K., WOLLISH, W., SOUTHWELL, B., LEECH, J. H. \& BROWN, G. V. (1991). Antigenic variation in Plasmodium falciparum. Proceedings of the National Academy of Sciences, USA 88, 9171-9174.

BRANNAN, L. R., TURNER, C. M. \& PHILLIPS, R. S. (1994). Malaria parasites undergo antigenic variation at high rates in vivo. Proceedings of the Royal Society of London, B 256, 71-75.

BUll, P. C., LOWE, B. S., KORTOK, M., MOLYNEUX, C. S., NEWBOLD, C. I. \& MARSH, K. (1998). Parasite antigens on the infected red cell surface are targets for naturally acquired immunity to malaria. Nature Medicine 4, 358-360.

CARLSON, J., Helmby, H., Hill, A. V., BREWSTER, D., GREENWOOD, B. M. \& WAHLGREN, M. (1990). Human cerebral malaria: association with erythrocyte rosetting and lack of anti-rosetting antibodies. Lancet 336, 1457-1460.

CHEN, Q., FERNANDEZ, V., SUNDSTROM, A., SCHLiCHTHERLE, M., DATa, S., Hagblom, P. \& WAHLGRen, M. (1998). Developmental selection of var gene expression in Plasmodium falciparum. Nature, London 394, 392-395.

Cheng, Q., LaWrence, G., REed, C., STOWERS, A., RANFORD-CARTWRight, L., CREASEy, A., CARTER, R. \& SAUl, A. (1997). Measurement of Plasmodium falciparum growth rates in vivo: a test of malaria vaccines. American Fournal of Tropical Medicine and Hygiene 57, 495-500.

COATney, G. R., COllins, W. E., Warren, M. \& CONTACos, P. G. (1971). The Primate Malarias. US Government Printing Office, Washington DC.

COLlins, W. E. \& JEFFERY, G. M. (1999). A retrospective examination of the patterns of recrudescence in patients infected with Plasmodium falciparum. American Fournal of Tropical Medicine and Hygiene 61, 44-48.

FRIED, M. \& DUFFY, P. E. (1996). Adherence of Plasmodium falciparum to chondroitin sulfate $\mathrm{A}$ in the human placenta. Science 272, 1502-1504.

GARDNER, J. P., PINCHES, R. A., ROBERTS, D. J. \& NEWBOLD, C. I. (1996). Variant antigens and endothelial receptor adhesion in Plasmodium falciparum. Proceedings of the National Academy of Sciences, USA 93, 3503-3508.

HANDUNNeTti, s. M., MENDis, K. N. \& DAVID, P. H. (1987). Antigenic variation of cloned Plasmodium fragile in its natural host Macaca sinica. Sequential appearance of successive variant antigenic types. Fournal of Experimental Medicine 165, 1269-1283.

IQBAL, J., SIRIPOON, N., SNOUNOU, V. G., PERLMANN, P. \& BERZINs, K. (1997). Plasmodium falciparum: selection 
of parasite subpopulations with decreased sensitivity for antibody-mediated growth inhibition in vitro.

Parasitology 114, 317-324. .

LAWrence, G., Cheng, Q., ReEd, C., TAYlor, D., STOWERS, A., ClOONAN, N., RZEPCZYK, C., SMillie, A., ANDERSON, K., POMBo, D., ALLWORTH, A., EISEN, D., ANDERS, R. \& SAUL, A. (2000). Effect of vaccination with 3 recombinant asexual-stage malaria antigens on initial growth rates of Plasmodium falciparum in non-immune volunteers. Vaccine 18, 1925-1931.

MOLINEAUX, L., DIEBNER, H. H., EICHNER, M., COLlins, W. E., JEFFERY, G. M. \& DiETZ, K. (2001). Plasmodium falciparum parasitaemia described by a new mathematical model. Parasitology 122, 379-391.

MOLINEAUX, L. \& DIETZ, K. (1999). Review of intra-host models of malaria. Parassitologia 41, 221-231.

NEWBOLD, C., CRAIG, A., KYES, S., ROWE, J. A., FERNANDEZREYES, D. \& FAGAN, T. (1999). Cytoadherence, pathogenesis and the infected red cell surface in Plasmodium falciparum. International Fournal for Parasitology 29, 927-937.

Reventos-Suarez, C., KaUl, D. K., MaCaluso, F. \& Nagel, R. L. (1985). Membrane knobs are required for the microcirculatory obstruction induced by Plasmodium falciparum-infected erythrocytes. Proceedings of the National Academy of Sciences, USA 82, 3829-3833.
ROBERTS, D., BIGGS, B. A., BROWN, G. \& NEWBOLD, C. (1993). Protection, pathogenesis and phenotypic plasticity in Plasmodium falciparum malaria.

Parasitology Today 9, 281-286.

Roberts, D. J., CRAIG, A. G., BERENDT, A. R., PINCHES, R., NASH, G., MARSH, K. \& NEWBOLD, C. I. (1992). Rapid switching to multiple antigenic and adhesive phenotypes in malaria. Nature, London 357, 689-692. ROWE, J. A., OBEIRO, J., NEWBOLD, C. I. \& MARSH, K.

(1995). Plasmodium falciparum rosetting is associated with malaria severity in Kenya. Infection and Immunity 63, 2323-2326.

SAUL, A. (1999). The role of variant surface antigens on malaria-infected red blood cells. Parasitology Today 15, 455-457.

SU, X. Z., Heatwole, v. M., WERTheimer, s. P., GUinet, F., HERRFELDT, J. A., PETERSON, D. S., RAVETCH, J. A. \& WELlEMs, T. E. (1995). The large diverse gene family var encodes proteins involved in cytoadherence and antigenic variation of Plasmodium falciparum-infected erythrocytes. Cell 82, 89-100.

TAYLOR, H. M., KYES, S., HARRIS, D., KRIEK, N. \& NEWBOLD, C. (2000). A study of var gene transcription in vitro using universal var gene primers. Molecular and Biochemical Parasitology 105, 13-23. 